\title{
Efficacy of Selective Nerve Root Block as a Diagnostic Tool in Radiculopathic Patients
} \author{
of Korea \\ Corresponding author: \\ Hyoung-Joon Chun \\ Department of Neurosurgery, \\ Hanyang University Medical \\ Center, Hanyang University \\ College of Medicine, 222-1, \\ Wangsimni-ro, Seongdong-gu, \\ Seoul 04763, Republic of Korea \\ Tel: $+82-2-2290-8492$ \\ Fax: +82-2-2281-0954 \\ E-mail: tdy815@hanyang.ac.kr
}

Sae Min Kwon ${ }^{1}$, Hyoung-Joon Chun ${ }^{2}$

${ }^{1}$ Departments of Neurosurgery, Asan Medical Center, University of Ulsan College of Medicine, Seoul, ${ }^{2}$ Department of Neurosurgery, Hanyang University Medical Center, Hanyang University College of Medicine, Seoul, Republic

Received: June 24, 2018 Revised: July 15, 2018 Accepted: July 16, 2018
Objective: Selective nerve root block (SNRB) is used for the diagnosis and treatment of different spinal disorders. However, the effectiveness of SNRB as a diagnostic tool is unclear. In this study, the efficacy of SNRB as a diagnostic tool for lumbar radiculopathy was evaluated. Methods: A total of 154 patients who underwent surgery for degenerative lumbar spine disease between March 2009 and February 2016 were enrolled in the present study. Patients were divided into two groups (with or without preoperative SNRB). The postoperative outcomes were evaluated using Odom's criteria at 30 days after surgery. Clinical and radiological findings were compared between the two groups. Results: Among the 154 patients, 52 (33.8\%) were included in the SNRB group. The patients in the SNRB group tended to have improved outcomes after surgery compared with those in the non-SNRB group $(p=0.077)$. In addition, the proportion of patients with favorable outcomes was significantly greater in the SNRB group ( $88.5 \%$ vs. $72.5 \%, p=0.041)$. Conclusion: SNRB is an excellent diagnostic tool for evaluating spinal disorders with radicular pain.

Key Words: Selective nerve root block; Diagnosis; Radiculopathy

\section{INTRODUCTION}

Currently, various preoperative studies including selective nerve root block (SNRB) and electromyography (EMG) have been used as diagnostic tools for lumbar radicular pain before surgery. Notably, SNRB is commonly used for diagnosis and treatment of spinal back pain or radiculopathy ${ }^{5)}$. Many studies have reported its value in the preoperative assessment of patients with radicular pain ${ }^{3,10,17)}$. SNRB and EMG are used for localizing anatomic sources of pain when clinical or radiological studies are equivocal. However, the application of SNRB as a diagnostic tool has not shown consistent efficacy. In the present study, we analyzed patients who underwent spinal surgery for radicular pain to identify the efficacy of $\mathrm{SNRB}$ as a preoperative diagnostic tool.

\section{MATERIALS AND METHODS}

\section{Patient Selection and Data Acquisition}

A retrospective single-center review of 199 consecutive patients who underwent surgery for degenerative lumbar spine disease between March 2009 and February 2016 was conducted.
Inclusion criteria for this study were patients who underwent surgery for degenerative spine disease including herniated lumbar disc, lumbar spinal stenosis, or lumbar spondylolisthesis. Among the 199 patients, 154 were included in the study. The remaining 45 patients were excluded for the following reasons: previous spine surgery $(n=25)$, postoperative infection $(n=6)$, postoperative hematoma $(n=4)$, or lost to follow-up $(n=4)$. Patients who underwent repeat surgery due to instrument malposition, cerebrospinal fluid leakage, or wound problems $(n=5)$ or who developed severe medical complications $(n=1)$ were also excluded.

All medical records from patients (hospital charts and radiographic studies) were collected. The demographic data, medical histories, and clinical and radiographic features were reviewed to evaluate the efficacy of SNRB as a diagnostic tool. The postoperative outcome was evaluated using the Odom's criteria (Table 1) at 30 days after surgery. According to the criteria, excellent or good outcome was considered favorable. Outcome measurements were performed at outpatient visits.

All patients were divided into two groups: patients with preoperative SNRB (SNRB group) or patients without SNRB (non-SNRB group). Clinical and radiological findings were compared between the 2 groups.

This study was approved by the Institutional Review Board (IRB) of Hanyang University Medical Center (IRB no. HYUH 
2017-12-021). Due to the retrospective nature of the study, the need for informed consent was waived.

\section{Technical Procedure}

All participants underwent SNRB by one neurosurgeon. The SNRB injection was fluoroscopically guided and generally performed with a 23-gauge, approximately $9 \mathrm{~cm}$-long (3.5-inch) spinal needle. Nonionic radiopaque myelographic contrast material was slowly instilled through the needle to reveal the outline of a target nerve root without evidence of vascular uptake. Subsequently, the injection solution was instilled slowly through the needle, and then the needle was removed. All participants were treated with a solution mixture of lidocaine, triamcinolone acetonide, and saline. A mixture including $20 \mathrm{~mL}$ of $2 \%$ lidocaine, $20 \mathrm{~mL}$ of saline, and $1 \mathrm{~mL}$ of triamcinolone acetonide $(40 \mathrm{mg} / \mathrm{mL}$ ) was prepared; $2 \mathrm{~mL}$ of the mixture was injected at each level (Fig. 1).

The test is considered positive for a given spinal nerve if needle contact produces pain similar to the patient's usual pain and if relief is experienced following local anesthetic injection, including a lack of pain during maneuvers that produced pain before the block, such as straight leg raising or walking.

After the injection procedure, the patient's skin was cleansed, and an adhesive bandage was placed on the puncture site.

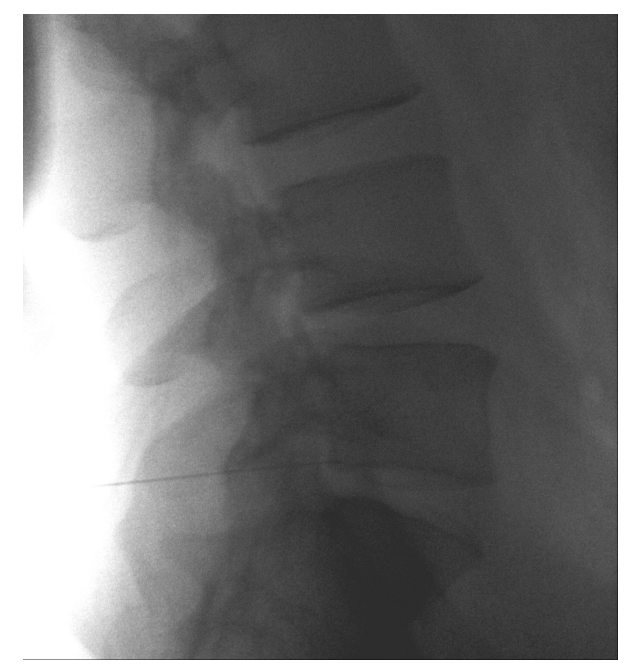

Fig. 1. Spinal needle is placed near the target nerve for selective nerve root block.
The patients were allowed to move and were reevaluated for evidence of motor loss.

\section{Statistical Analysis}

All statistical analyses were performed using SPSS version 18.0 (SPSS Inc., Chicago, IL, USA). Student's $t$ test or the MannWhitney U test was used for comparison of continuous variables, and $X^{2}$ or Fisher's exact test was used for the comparison of categorical variables. A p-value $<0.05$ was considered statistically significant in all analyses.

\section{RESULTS}

A total of 154 patients (82 women; $53.5 \pm 17.1$ years of age) met the criteria. Of these, $52(33.8 \%)$ underwent preoperative $\mathrm{SNRB}$ as a diagnostic tool. Table 2 shows the baseline characteristics of the enrolled patients. Demographics, medical history, length of hospital stay, diagnosis, number of affected levels, and surgical time were not significantly different between the groups. Although the results did not satisfy statistical significance, patients in the SNRB group had more excellent ( $42.3 \%$ vs. $36.3 \%)$ or good (46.2\% vs. $36.3 \%)$ grade and less fair $(11.5 \%$ vs. $27.5 \%$ ) grade compared with those in the non-SNRB group. A poor grade was not observed in any patient from either group. In terms of favorable outcome (excellent or good grade), the SNRB group showed significantly better results (88.5\% vs. $72.5 \%, \mathrm{p}=0.041)$.

Table 3 shows the results when comparing only patients with two or more levels. In patients with multiple levels, there was a difference in postoperative outcome between the 2 groups. However, due to the small number of patients, this result was not statistically significant.

\section{DISCUSSION}

SNRB, when combined with a careful physical examination and quality radiologic studies, is an important tool in the diagnostic evaluation of patients with predominantly radicular symptoms ${ }^{6}$. SNRB may be used to define the source of pain and is especially useful when clinical examinations, electrodiagnostic studies, and imaging studies are equivocal ${ }^{11)}$. Diagnostic nerve root blocks may be particularly useful to identify

Table 1. Odom's criteria

\begin{tabular}{ll}
\hline \hline \multicolumn{1}{c}{ Grading } & Definition \\
\hline Excellent & All preoperative symptoms relieved; abnormal findings improved \\
Good & Minimal persistence of preoperative symptoms; abnormal findings unchanged or improved \\
Fair & Definite relief of some preoperative symptoms; other symptoms unchanged or slightly improved \\
Poor & Symptoms and signs unchanged or exacerbated \\
\hline
\end{tabular}


Table 2. Baseline characteristics of patients

\begin{tabular}{|c|c|c|c|c|}
\hline Variable & Total $(n=154)$ & SNRB group $(\mathrm{n}=52)$ & Non-SNRB group $(n=102)$ & p-value \\
\hline Age (years) & $53.5 \pm 17.1$ & $52.4 \pm 17.4$ & $54.0 \pm 17.0$ & 0.572 \\
\hline Sex (female) & $82(53.2 \%)$ & $24(46.2 \%)$ & 58 (56.9\%) & 0.276 \\
\hline \multicolumn{5}{|l|}{ Medical history } \\
\hline Hypertension & 49 (31.8\%) & 14 (26.9\%) & 35 (34.3\%) & 0.454 \\
\hline Diabetes mellitus & $14(9.1 \%)$ & $7(13.5 \%)$ & $7(6.9 \%)$ & 0.293 \\
\hline Other comorbidities & $14(9.1 \%)$ & $4(7.7 \%)$ & $10(9.8 \%)$ & 0.893 \\
\hline BMI & $23.6 \pm 3.4$ & $23.0 \pm 2.7$ & $24.0 \pm 3.8$ & 0.231 \\
\hline Length of hospital stay (day) & $12(10-14)$ & $12(10-14.5)$ & $12(10-14)$ & 0.970 \\
\hline Diagnosis & & & & 0.484 \\
\hline HLD & $104(67.5 \%)$ & $34(65.4 \%)$ & $70(68.6 \%)$ & \\
\hline Spinal stenosis & 31 (20.1\%) & $13(25.0 \%)$ & $18(17.6 \%)$ & \\
\hline Spondylolisthesis \pm spinal stenosis & 19 (12.3\%) & $5(9.6 \%)$ & $14(13.7 \%)$ & \\
\hline Total levels & & & & 0.793 \\
\hline 1 level & $129(83.8 \%)$ & 45 (86.5\%) & 84 (82.4\%) & \\
\hline 2 levels & 21 (13.6\%) & $6(11.5 \%)$ & $15(14.7 \%)$ & \\
\hline 3 levels & $4(2.6 \%)$ & $1(1.9 \%)$ & $3(2.9 \%)$ & \\
\hline Surgical time (minutes) & $135(100-162.5)$ & $120(97.5-147.5)$ & $140(105-175)$ & 0.126 \\
\hline Odom's criteria & & & & 0.077 \\
\hline Excellent & 59 (38.3\%) & $22(42.3 \%)$ & $37(36.3 \%)$ & \\
\hline Good & 61 (39.6\%) & $24(46.2 \%)$ & 37 (36.3\%) & \\
\hline Fair & $34(22.1 \%)$ & $6(11.5 \%)$ & 28 (27.5\%) & \\
\hline Poor & $0(0.0 \%)$ & 0 (0.0\%) & 0 (0.0\%) & \\
\hline Favorable outcome & 120 (77.9\%) & $46(88.5 \%)$ & $74(72.5 \%)$ & 0.041 \\
\hline
\end{tabular}

BMl: body mass index; HLD: herniated lumbar disc; SNRB: selective nerve root block.

Table 3. Comparison between selective nerve root block and non-selective nerve root block groups in patients with multi-level involvement

\begin{tabular}{lccc}
\hline \hline Variable & SNRB group $(\mathrm{n}=7)$ & Non-SNRB group $(\mathrm{n}=18)$ & $\mathrm{p}$-value \\
\hline Age (years) & $66.0 \pm 6.9$ & $60.8 \pm 16.9$ & 0.285 \\
Sex (female) & $5(71.4 \%)$ & $10(55.6 \%)$ & 0.785 \\
BMl & $23.8 \pm 2.5$ & $25.2 \pm 4.5$ & 0.629 \\
Length of hospital stay (day) & $13.0(12.5-15.5)$ & $12.5(12.0-14.0)$ & 0.357 \\
Diagnosis & & & 0.944 \\
$\quad$ HLD & $1(14.3 \%)$ & $3(16.7 \%)$ & \\
Spinal stenosis & $4(57.1 \%)$ & $11(61.1 \%)$ & \\
Spondylolisthesis \pm spinal stenosis & $2(28.6 \%)$ & $4(22.2 \%)$ & \\
Preoperative EMG & $3(42.9 \%)$ & $4(22.2 \%)$ & 0.592 \\
Surgical time (minutes) & $95.0 \pm 10.0$ & $107.8 \pm 19.2$ & 0.306 \\
Odom's criteria & & & 0.212 \\
$\quad$ Excellent & $2(28.6 \%)$ & $3(16.7 \%)$ & \\
Good & $5(71.4 \%)$ & $9(50.0 \%)$ & \\
Fair & $0(0.0 \%)$ & $6(33.3 \%)$ & \\
$\quad$ Poor & $0(0.0 \%)$ & $0(0.0 \%)$ & \\
Favorable outcome & $7(100.0 \%)$ & $12(66.7 \%)$ & 0.218 \\
\hline
\end{tabular}

BMl: body mass index; HLD: herniated lumbar disc; EMG: electromyography; SNRB: selective nerve root block.

34 www.thenerve.net 
the symptomatic level in patients with multi-level pathology, ${ }^{1,4)}$. Similarly, SNRB may also be useful when the location of symptoms appears to conflict with abnormalities identified in radiologic findings. In the present study, although not statistically significant, postoperative outcomes defined according to Odom's criteria were better in the SNRB group than in the non-SNRB group. Moreover, the proportion of patients with favorable outcome was significantly greater in the SNRB group than in the non-SNRB group.

EMG has limitations in localizing the patient's radicular pain to a single level ${ }^{15)}$. In addition, radiologic studies may be difficult to interpret after spinal surgery due to scar tissue in the epidural space and other anatomic changes ${ }^{14)}$. Furthermore, radicular symptoms may not correspond to classic dermatomal patterns ${ }^{13)}$. The rationale for a diagnostic SNRB is that, if a particular spinal nerve is responsible for causing or mediating patient symptoms, then anesthetizing the nerve should temporarily relieve symptoms. If the symptom is pain, anesthetization of the responsible nerve should relieve the pain. If the symptom is paresthesia, anesthetizing the responsible nerve should produce numbness in the area where the paresthesia was previously present. If the symptom is numbness, anesthetizing the responsible nerve should produce no change in the numbness or perhaps accentuate it. If a nerve anesthetized is not responsible for the patient's symptoms, pain will not be relieved, and numbness in the distribution will occur ${ }^{2)}$.

The accuracy and effectiveness of SNRB as a diagnostic test have been reported in several studies. Haueisen et al. ${ }^{8)}$ reported on patients with sciatica of unclear etiology who underwent SNRB; an accurate diagnosis was made in 43 of $46(93 \%)$ patients. Schutz et al. ${ }^{12)}$ retrospectively reported on SNRBs performed in 23 patients. In 15 patients, an operation was performed at the level indicated by SNRB, and the operative findings were in agreement with SNRB in $13(87 \%)$ patients ${ }^{12)}$.

In contrast, there are confounding variables that can undermine the validity of our study. Injection of local anesthetics may spread beyond the intended spinal nerve target to structures such as adjacent dorsal rami, spinal nerves, or the sinuvertebral nerves, thereby causing a false-positive result. Anatomical variation is another potential problem. In a cadaver study, nerve root abnormalities were found in $14 \%$ of individuals ${ }^{9}$. The furcal nerve usually arises from the L4 root level and contributes to both the lumbar and sacral plexuses of nerves ${ }^{7}$. Neurological symptoms, suggestive of 2 roots being involved, frequently result from furcal nerve compression.

Pain is purely subjective, often with uncertain pathophysiology, and may be influenced by psychological, social, financial, and legal factors, as well as by the efficacy of concurrent therapies such as medications and physical therapy. Additionally, spinal injections may be associated with a significant placebo effect. Reportedly, the placebo effect increased in direct correlation to the invasiveness of a procedure ${ }^{16)}$.

The present study had several limitations. First, our data were retrospectively analyzed. In addition, the number of patients included in our study was rather small, which limits the statistical power to appropriately differentiate the relative importance of diagnostic tests studied. Finally, the pain-blocking effect of SNRB can influence the postoperative prognosis. Moreover, the above-mentioned confounding variables render the procedure difficult to precisely perform.

\section{CONCLUSION}

In the present study, the patients who underwent SNRB preoperatively had better prognosis after surgery than patients who did not. Although the statistical significance was not satisfied, further studies are necessary with large series.

\section{CONFLICT OF INTEREST}

No potential conflict of interest relevant to this article was reported.

\section{REFERENCES}

1. Anderberg L, Annertz M, Rydholm U, Brandt L, S?veland H: Selective diagnostic nerve root block for the evaluation of radicular pain in the multilevel degenerated cervical spine. Eur Spine J 15:794-801, 2006

2. Datta S, Everett CR, Trescot AM, Schultz DM, Adlaka R, Abdi S, et al.: An updated systematic review of the diagnostic utility of selective nerve root blocks. Pain Physician 10:113-128, 2007

3. Datta S, Manchikanti L, Falco FJ, Calodney AK, Atluri S, Benyamin RM, et al.: Diagnostic utility of selective nerve root blocks in the diagnosis of lumbosacral radicular pain: systematic review and update of current evidence. Pain Physician 16:Se97124, 2013

4. Eastley NC, Spiteri V, Newey ML: Variations in selective nerve root block technique. Ann R Coll Surg Engl 95:515-518, 2013

5. Eckel TS, Bartynski WS: Epidural steroid injections and selective nerve root blocks. Tech Vasc Interv Radiol 12:11-21, 2009

6. Everett CR, Shah RV, Sehgal N, McKenzie-Brown AM: A systematic review of diagnostic utility of selective nerve root blocks. Pain Physician 8:225-233, 2005

7. Harshavardhana NS, Dabke HV: The furcal nerve revisited. Orthop Rev (Pavia) 6:5428, 2014

8. Haueisen DC, Smith BS, Myers SR, Pryce ML: The diagnostic accuracy of spinal nerve injection studies. Their role in the evaluation of recurrent sciatica. Clin Orthop Relat Res:179-183, 1985

9. Kadish LJ, Simmons EH: Anomalies of the lumbosacral nerve roots. An anatomical investigation and myelographic study. J Bone Joint Surg Br 66:411-416, 1984

10. North RB, Kidd DH, Zahurak M, Piantadosi S: Specificity of diagnostic nerve blocks: a prospective, randomized study of sciatica due to lumbosacral spine disease. Pain 65:77-85, 1996 
11. Sasso RC, Macadaeg K, Nordmann D, Smith M: Selective nerve root injections can predict surgical outcome for lumbar and cervical radiculopathy: comparison to magnetic resonance imaging. J Spinal Disord Tech 18:471-478, 2005

12. Schutz H, Lougheed WM, Wortzman G, Awerbuck BG: Intervertebral nerve-root in the investigation of chronic lumbar disc disease. Can J Surg 16:217-221, 1973

13. Slipman CW, Plastaras CT, Palmitier RA, Huston CW, Sterenfeld EB: Symptom provocation of fluoroscopically guided cervical nerve root stimulation. Are dynatomal maps identical to dermatomal maps? Spine (Phila Pa 1976) 23:2235-2242, 1998

14. Stanley D, McLaren MI, Euinton HA, Getty CJ: A prospective study of nerve root infiltration in the diagnosis of sciatica. A comparison with radiculography, computed tomography, and operative findings. Spine (Phila Pa 1976) 15:540-543, 1990

15. Tullberg T, Svanborg E, Isaccsson J, Grane P: A preoperative and postoperative study of the accuracy and value of electrodiagnosis in patients with lumbosacral disc herniation. Spine (Phila Pa 1976) 18:837-842, 1993

16. Turner JA, Deyo RA, Loeser JD, Von Korff M, Fordyce WE: The importance of placebo effects in pain treatment and research. JAMA 271:1609-1614, 1994

17. Yeom JS, Lee JW, Park KW, Chang BS, Lee CK, Buchowski JM, et al.: Value of diagnostic lumbar selective nerve root block: a prospective controlled study. AJNR Am J Neuroradiol 29: 1017-1023, 2008 